\title{
Analysis of Actuator Faults in a Cooperative Team Consensus of Unmanned Systems
}

\author{
E. Semsar-Kazerooni and K. Khorasani
}

\begin{abstract}
This work presents results on performance analysis of a team of agents in the presence of team members faults for three types of faults, i.e. loss of effectiveness, float, and lock-in-place faults. The team goal is to accomplish a cohesive motion in a modified leader-follower architecture using a semidecentralized optimal control. This controller that is recently proposed by the authors is designed based on minimization of individual cost functions using local information. It is shown that a loss of effectiveness (LOE) fault does not deteriorate the stability or the consensus seeking goal of the team and would only result in a different transient behavior, e.g. a change in the agent's convergence rate, without a change in the consensus value. On the other hand if the fault in one or more of the agents is of the float type, either in the leader or the followers, the team does not maintain its consensus anymore but stability of the team can be guaranteed. Moreover, the leader and the healthy followers adapt themselves to the followers changes when a float fault occurs in one of the agents. Finally, the behavior of the team in the presence of a lock-in-place (LIP) fault is also discussed. Simulation results are provided to demonstrate the performance of the team in the presence of the above faults.
\end{abstract}

\section{INTRODUCTION}

Enhanced group robustness to individual failures, increased and improved instrument sensing and resolution, and adaptive reconfigurability capabilities are among the motivating advantages for deploying autonomous network of unmanned systems [1]. Although a large body of work has been developed to address the requirements of design and utilization of these networks, there are still many unsolved problems in this domain, (see [2]-[7] for the recent literature reviews on the subject). One of the main problems that arises in development of an optimal cooperation in a team of unmanned systems is lack of complete information and presence of uncertainties, faults and unpredictable events in the team. In [8], [9] leader commands are assumed to be unknown for the follower, and therefore an adaptive controller is used for formation keeping.

In practice, it is quite possible that some agents in the team may be unable to follow the team command due to their anomalies and faults. Some sources of this problem can be due to actuator faults or saturation, faults in measurement of neighbors states, or communication links faults. Due to these malfunctions, the faulty agent cannot follow the command provided by the team to achieve the predefined goal. This may result in permanent separation of that agent from the team which correspondingly may affect the cohesion of the team. Here, we consider three types of actuator faults, i.e.

The authors are with the Department of Electrical and Computer Engineering, Concordia University, 1455 de Maisonneuve Blvd. W., Montreal, Quebec, H3G 1M8, Canada, (e-mails: e_semsar, kash@ece.concordia.ca). loss of effectiveness, float, and lock-in-place faults. In loss of effectiveness, the applied control signal is a portion of the designed control signal, whereas in the float fault the control signal vanishes at some point. In lock-in-place fault, the control signal is freezed at some point and does not change anymore. In [6], the behavior of the team when a float fault is injected in some of the team members is studied. The cooperative team design is based on a semi-decentralized optimal control strategy [10]. It was shown that in case of faults in one or more agents, whether it is in the leader or the followers, the team maintains its stability. Also, the final steady state value to which the team would converge is derived and predicted. In this situation the leader's behavior is shown to be adaptable to that of the faulty agent, e.g. in case of a follower speed reduction, the leader would also correspondingly decrease its speed.

The main contribution of the present work is to formally analyze the team behavior in presence of actuator anomalies and faults. First, for a modified leader-follower (MLF) team of agents the effects of a loss of effectiveness (LOE) fault is discussed. It is shown that stability and consensus achievement are maintained under this situation. Next, we extend the work of [6] where, for a float type of fault, the followers are shown to be adaptable to changes that occur in the faulty agent. The leader adaptability was shown and verified formally in [6] and here we proved the same property for the followers. In this manner cohesion and cooperation of the team is maintained and the team remains together until the fault is recovered. Finally, the behavior of the team in the presence of a lock-in-place (LIP) fault is investigated.

The organization of this paper is as follows: In Section 2, problem formulation is given. In Section 3, the team behavior for a LOE scenario is analyzed. In Section 4, analysis of the team behavior in case of the float fault is provided and in Sections 5 the effect of LIP fault on team performance is investigated. Finally, in Sections 6 and 7 simulation results and conclusions are discussed, respectively.

\section{BACKGROUND PRELIMINARIES}

Multi-Agent Teams: Assume a set of agents, e.g. mobile robots, $\Omega=\{i \mid i=1, \ldots, N\}$, where $N$ is the number of agents in a team. In this paper two dynamical models that are associated with an agent are considered. The first model is a linear model of an agent or a vehicle that is given by

$$
\left\{\begin{array}{l}
\dot{r}^{i}=\bar{A}^{i} v^{i} \\
\dot{v}^{i}=A^{i} v^{i}+B^{i} u^{i} \\
u^{i}:=u_{l}^{i}+u_{g}^{i}, u_{g}^{i}=B^{i} \sum_{j \in N^{i}} F^{i j} Y^{j} \\
Y^{i}=v^{i}, i=1, \ldots, N
\end{array}\right.
$$


The second representation is a double integrator model which is a special case of this model by replacing $\bar{A}^{i}=I, A^{i}=$ $0, B^{i}=I$, i.e.

$$
\left\{\begin{array}{l}
\dot{r^{i}}=v^{i} \\
\dot{v^{i}}=u^{i}:=u_{l}^{i}+u_{g}^{i}=u_{l}^{i}+\sum_{j \in N^{i}} F^{i j} v^{j}
\end{array}\right.
$$

where $r^{i}, v^{i} \in R^{m}$ are the position and velocity vectors, and $u^{i}$ and $Y^{i}$ are the input and the output vectors, respectively. The input signal $u^{i}$ is decomposed into $u_{l}^{i}$ and $u_{g}^{i}$ that represent the "local" and the "global" control terms, respectively [10]. The local term for each agent is designed using the agent's own output vector, whereas the global control utilizes the information received from other agents in the neighboring set of the considered agent. The "global" control term $u_{g}^{i}\left(Y^{j}\right)=\sum_{j \in N^{i}} F^{i j} v^{j}$ is also denoted as the interaction term, where $F^{i j}$ is the interaction matrix to ensure compatibility in the agent's input and output channels dimensions. Note that for the purpose of our formal analysis and development it is sufficient to consider only the velocity dynamics and the position dynamics is used simply for simulation studies.

Information Structure and Neighboring Sets: In order to ensure cooperation and coordination among team members, each agent has to know the status (defined as the agent's output) of other members, and therefore, it has to communicate with others. In an information structure described with an undirected graph, the set of agents connected to agent $i$ via communication links is called a neighboring set $N^{i}$, that is, $\forall i=1, \ldots, N, \quad N^{i}=\{j=1, \ldots, N \mid j \mathcal{R} i\}$, in which $\mathcal{R}$ designates that agents $i$ and $j$ have either a communication link to transfer their status to each other or a means to measure their status or exchange information with one another.

Modified Leader-Follower (MLF) Structure: Assume an information exchange structure where an external command is provided to one of the members designated as the leader, and the goal is to make the agents' output, e.g. velocity, converge to a desired specified value, i.e. $Y^{i} \rightarrow Y^{d}, \forall i$. Other agents should follow the leader by communicating through their links with each other and with the leader. Furthermore, the leader has two-way links with the followers that are connected to it.

Actuator Fault Types: In [11], three types of actuator faults are introduced, namely i) Lock-In-Place (LIP), ii) Float, and iii) Loss of Effectiveness (LOE). These faults may be formally represented as follows,

$$
\begin{aligned}
& u_{f}^{i}= \\
& \left\{\begin{array}{lll}
\alpha u^{i} & 0<\epsilon<\alpha<1, \forall t \geq t_{f} & \text { Loss of } \\
0 & \alpha=1, \forall t \geq t_{f} & \text { Effectiveness } \\
u^{i}\left(t_{f}\right) & \alpha=1, \forall t \geq t_{f} & \text { Lloat }
\end{array}\right.
\end{aligned}
$$

where $u_{f}^{i}$ corresponds to the actual input produced by an actuator, $u^{i}$ is the input commanded by the controller, $t_{f}$ denotes the time when a fault is injected, and $\alpha$ represents the effectiveness coefficient of the actuator which is defined to be $\alpha \in[\epsilon, 1], \epsilon>0$.

A review on semi-decentralized optimal control design for team cooperation seeking: Design of semi-decentralized optimal control strategies is discussed in [10] in detail. In this section, we briefly present the required results. The main goal in [10] is to make the agents' output converge to a common value, i.e. $Y^{i} \rightarrow Y^{j} \rightarrow Y^{d}, \forall i, j$ which is enforced from outside and is provided to the leader. The output variable can be velocity, position, or any other state on which the team should have a consensus. Towards this end, let us define the cost functions for the follower agents as

$$
\begin{gathered}
d^{i}=\int_{0}^{T}\left\{\sum_{j \in N^{i}}\left[\left(Y^{i}-Y^{j}\right)^{T} Q^{i j}\left(Y^{i}-Y^{j}\right)\right]+\right. \\
\left.\left(u^{i}\right)^{T} R^{i} u^{i}\right\} d t
\end{gathered}
$$

The leader's cost is selected according to

$$
\begin{aligned}
d^{1} & =\int_{0}^{T}\left\{\sum_{j \in N^{1}}\left[\left(Y^{1}-Y^{j}\right)^{T} Q^{1 j}\left(Y^{1}-Y^{j}\right)\right]\right. \\
& \left.+\left[\left(Y^{1}-Y^{d}\right)^{T} \Gamma\left(Y^{1}-Y^{d}\right)\right]+\left(u^{1}\right)^{T} R^{1} u^{1}\right\} d t(5)
\end{aligned}
$$

where the superscript 1 is used to denote the leader and $Q^{i j}, \Gamma$ and $R^{i}$ are symmetric and positive definite matrices.

Lemma 1 a) Modified Consensus Protocol: Assume a team of agents whose dynamics are governed by (2) and having an MLF structure. Associated with the infinite horizon scenario (i.e., $T \longrightarrow \infty$ ), the control laws proposed below would ensure a solution to the corresponding HamiltonJacobi-Bellman (HJB) equations and simultaneously minimize the cost functions (4) and (5). Furthermore, this will guarantee consensus among and alignment of the agents. The control protocol for the follower is given by

$$
u^{i}\left(v^{i}, v^{j}\right)=u_{l}^{i}\left(v^{i}\right)+u_{g}^{i}\left(v^{j}\right)=\Gamma^{i}\left(v^{i}-\frac{\sum_{j \in N^{i}} v^{j}}{\left|N^{i}\right|}\right)
$$

and for the leader is governed by

$$
u^{1}\left(v^{1}, v^{j}\right)=\Gamma^{1}\left(v^{1}-\frac{\sum_{j \in N^{1}} v^{j}}{\left|N^{1}\right|}\right)+\beta^{1}\left(v^{1}-v^{d}\right)
$$

where

$$
\begin{aligned}
& 2\left(\left|N^{1}\right| Q^{1 j}+\Gamma\right)-\frac{1}{2} K^{1}\left(R^{1}\right)^{-1} K^{1}=0, \\
& 2\left|N^{i}\right| Q^{i j}-\frac{1}{2} K^{i}\left(R^{i}\right)^{-1} K^{i}=0, i=2, \ldots, N \\
& \Gamma^{i}=-2\left(K^{i}\right)^{-1}\left|N^{i}\right| Q^{i j}, \forall i, \quad \beta^{1}=-2\left(K^{1}\right)^{-1} \Gamma
\end{aligned}
$$

b) Stability: The above control protocol for each agent is stabilizing, i.e. the error dynamics of the entire team is asymptotically stable, implying that

$$
e^{i}=v^{i}-v^{d} \rightarrow 0 \text { as } t \rightarrow \infty, \quad i=1, \ldots, N
$$

Proof: The details can be found in [10].

From (7), it follows that while the second term in the leader's control law guarantees command tracking, the first part maintains the team cohesion and guarantees that none 
of the followers is "lost" without affecting the others', and specifically the leader's behavior. This is an advantage of our proposed architecture when compared to the conventional leader-follower structure, where if a follower fails to follow the leader's command, it would be separated from the team and could be lost from the team forever. However, in our proposed structure if one of the followers cannot follow the command, all other agents would adapt themselves to this change until this agent is recovered. This property will be formalized in the following sections.

To conclude this section, similar results can be obtained if dynamical model (1) is considered for the team agents [12]. Specifically, we have the following lemma.

Lemma 2 a) Modified Consensus Protocol: Assume a team of agents whose dynamics are governed by (1) and having an MLF structure. Associated with the infinite horizon scenario, the following control laws would ensure a solution to the corresponding HJB equations and simultaneously minimize the cost functions (4) and (5). Furthermore, this will guarantee consensus among and alignment of the agents. The control protocol for a follower is given by

$$
u^{i}\left(v^{i}, v^{j}\right)=\Gamma^{i}\left(v^{i}-\frac{\sum_{j \in N^{i}} v^{j}}{\left|N^{i}\right|}\right)+\beta^{i} v^{i}, i=2, \ldots, N
$$

and for the leader is governed by

$$
\begin{aligned}
& u^{1}=\Gamma^{1}\left(v^{1}-\frac{\sum_{j \in N^{1}} v^{j}}{\left|N^{1}\right|}\right)+\alpha^{1}\left(v^{1}-v^{d}\right)+\beta^{1} v^{1} \\
& -\left(K^{1} B^{1}\right)^{-1}\left(A^{1}\right)^{T} g^{1}
\end{aligned}
$$

in which $\alpha^{1}=-2\left(K^{1} B^{1}\right)^{-1} \Gamma, \Gamma^{i}=-2\left(K^{i} B^{i}\right)^{-1}\left|N^{i}\right| Q^{i j}$, and $\beta^{i}=-\left(K^{i} B^{i}\right)^{-1}\left(K^{i} A^{i}+\left(A^{i}\right)^{T} K^{i}\right), \forall i$ and

$$
\begin{aligned}
& 2\left|N^{i}\right| Q^{i j}-\frac{1}{2} K^{i} B^{i}\left(R^{i}\right)^{-1}\left(B^{i}\right)^{T} K^{i}+\left(A^{i}\right)^{T} K^{i}+K^{i} A^{i} \\
& =0, i=2, \ldots, N \\
& 2\left(\left|N^{1}\right| Q^{1 j}+\Gamma\right)+\left(A^{1}\right)^{T} K^{1}+K^{1} A^{1} \\
& -\frac{1}{2} K^{1} B^{1}\left(R^{1}\right)^{-1}\left(B^{1}\right)^{T} K^{1}=0 \\
& 2 \Gamma v^{d}+\left(\frac{1}{2} K^{1} B^{1}\left(R^{1}\right)^{-1}\left(B^{1}\right)^{T}-\left(A^{1}\right)^{T}\right) g^{1}=0
\end{aligned}
$$

b) Stability: The above control protocol is stabilizing, i.e. the error dynamics of the entire team is asymptotically stable, implying that $e^{i}=v^{i}-v^{d} \rightarrow 0$ as $t \rightarrow \infty$, if matrices $\Gamma, K^{1}$, and $R^{1}$ are determined appropriately so that the following set of LMIs is satisfied

$$
\left\{\begin{array}{l}
\Upsilon+\Upsilon^{T}>0, \Upsilon=2 L \otimes Q^{i j}+A^{T} K+2 G \\
\left(A^{1}\right)^{T} K^{1}+K^{1} A^{1}+Z^{1}+2\left(\left|N^{1}\right| Q^{1 j}+\Gamma\right)=0 \\
Z^{1}=-\frac{1}{2} K^{1} B^{1}\left(R^{1}\right)^{-1}\left(B^{1}\right)^{T} K^{1} \\
\Gamma>0, K^{1}>0, Z^{1}<0
\end{array}\right.
$$

where $K=\operatorname{Diag}\left\{K^{i}, i=1, \ldots, N\right\}, A=\operatorname{Diag}\left\{A^{i}, i=\right.$ $1, \ldots, N\}, G=\operatorname{Diag}\{\Gamma, 0, \ldots, 0\}$, and $L$ is the Laplacian matrix of the network underlying graph.

Proof: The details can be found in [12].

\section{TEAM BEHAVIOR SUBJECT TO LOSS OF EFFECTIVENESS (LOE) IN SOME OF THE AGENTS' ACTUATORS}

For the team of agents described in Lemma 1, assume that some of the agents, either some of the followers or the leader, fail to produce the team control command as described in Lemma 1. In other words due to the actuator loss of effectiveness (LOE) fault one instead has $u_{f}^{i}=\alpha u^{i}, 0<$ $\alpha \leq 1$, where $u_{f}^{i}$ stands for the actual control effect that is applied by the actuator with $u^{i}$ representing the designed control input. Let us denote the set of failed agents by $\Omega_{f}=\{i=N-q+1, \ldots, N\}$, and without loss of generality assume that these are the last $q$ agents of the team. If this is not the case, the agents' labels can be easily changed for this purpose. Using the notion of error as introduced in Lemma 1, let us define the total error vector corresponding to the healthy agents as $e_{w}$ and the one corresponding to the faulty agents as $e_{f}$. Now, assume that the closed-loop dynamics of the entire team is described by $\dot{e}=L_{c l} e$, where $e=\left[\begin{array}{lll}\left(e^{1}\right)^{T} & \ldots & \left(e^{N}\right)^{T}\end{array}\right]^{T}=\left[\begin{array}{ll}e_{w}^{T} & e_{f}^{T}\end{array}\right]^{T}$. Partition $L_{c l}$ in order to separate the dynamics of the faulty and the healthy agents as follows

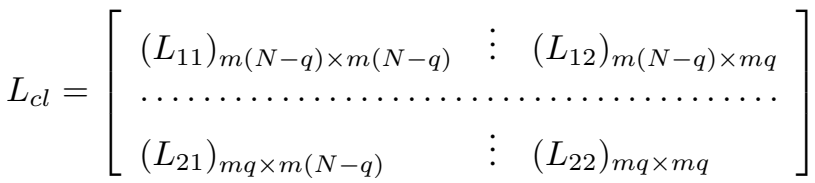

where $m$ is the dimension of $v^{i}$ (assumed here to be 2). Due to the presence of faults, the closed-loop error dynamics is now be modified to

$$
\dot{e}=\left[\begin{array}{ccc}
L_{11} & \vdots & L_{12} \\
\ldots \ldots & \ldots & \ldots \\
\alpha L_{21} & \vdots & \alpha L_{22}
\end{array}\right]\left[\begin{array}{l}
e_{w} \\
e_{f}
\end{array}\right]
$$

The following lemma shows that the error dynamics will remain stable despite the presence of agents' faults. Moreover, the consensus achieving goal can still be guaranteed.

Lemma 3 a) Stability Analysis: For the team of agents described in Lemma 1, if some of the agents fail to comply with the team control command as described in Lemma 1 and instead implement $u_{f}^{i}=\alpha u^{i}$ due to the LOE fault, the resulting closed-loop error dynamics still remains stable.

b) Consensus Achievement: Moreover, the error, i.e. $e^{i}=$ $v^{i}-v^{d}, \forall i$ will asymptotically approach to zero and hence the consensus will be achieved.

Proof: Omitted due to space limitations.

\section{TEAm BeHAVIOR SUbJECT TO AN ACTUATOR FLOAT FAULT}

For the team of agents described in Lemma 1, assume that a number of the follower agents fail to produce the team control command as described in (6), and due to the actuator "float" fault one now instead has $u^{i}=0$. Denote the set of failed agents by $\Omega_{f}$, the total error vector corresponding to the healthy agents by $e_{w}$, and the one corresponding to the 
faulty agents by $e_{f}$, as defined in the previous section. The concatenated velocity vector of failed agents can be defined as $v_{f}=\left[\left(v^{N-q+1}\right)^{T}, \ldots,\left(v^{N}\right)^{T}\right]^{T}$ which is a constant vector (due to $u^{i}$ being zero). In addition, $e_{f}$ would be a constant vector if $v^{d}$ is time-invariant. Now, assume that the entire team closed-loop error dynamics is described by $\dot{e}=$ $L_{c l}$ e, and let us partition $L_{c l}$ as before. Due to the presence of faults, the closed-loop error dynamics is now modified to

$$
\dot{e}=\left[\begin{array}{ccc}
L_{11} & \vdots & L_{12} \\
\ldots \ldots \ldots \ldots \ldots \ldots \ldots \ldots \\
0_{m q \times m(N-q)} & \vdots & 0_{m q \times m q}
\end{array}\right]\left[\begin{array}{c}
e_{w} \\
e_{f}
\end{array}\right]
$$

In [6], it is shown that the error dynamics will remain stable despite the presence of the followers faults. Similar results are obtained in [6] for the scenario when the fault occurs in the leader. There, it is shown that the healthy leader will adapt itself to the state of the faulty agent. Here, we prove the same property for the healthy followers.

Lemma 4 Leader and Followers Adaptability: For the team of agents described in Lemma 1, if a follower, i.e. $v_{f}=v^{N}$, fails to produce the team control command as specified in (6) due to a float fault, i.e. $u^{i}=0$, then all the agents will adapt themselves to this agent's change, i.e. the direction of change in the state of the faulty follower would be the same as the change in the rest of the team. This implies that the steady state error of the faulty follower and the healthy members will have the same sign, i.e.

$e^{k} \bullet e_{f}>0, e_{f}=v_{f}-v^{d}, e^{k}=v^{k}-v^{d}, k=1, \ldots, N-1$

in which $v_{f}$ is the velocity at which the faulty agent's velocity is frozen, "•" is the Hadamard product [13], and " $>0$ " refers to positiveness of vector's elements.

Proof: Omitted due to space limitations.

\section{TEAM BEHAVIOR SUBJECT TO LOCK-IN-PLACE (LIP) FAULT IN ONE OF THE AGENTS}

For the team of agents described in Lemma 1, assume that one of the followers fails to produce the team control command. In other words, due to a LIP failure the applied control input is frozen at a constant value, namely, $u_{f}^{i}=u_{c}$, where $u_{c}$ is a constant value. In this situation and similar to the discussions of the previous sections, the closed-loop dynamics reduces to

$$
\begin{aligned}
& \dot{e}=\left[\begin{array}{c}
\dot{e}_{w} \\
\dot{e}_{f}
\end{array}\right]=
\end{aligned}
$$

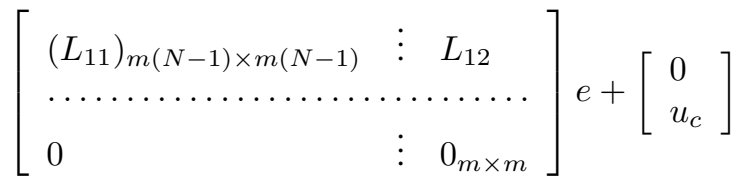

From the above equation it can be deduced that $e_{f}$ can grow without a bound, and therefore the error dynamics in (15) is not stable. In other words, to guarantee stability in this situation at least the open-loop matrix of the faulty agent should be stable. Towards this end, we first determine the agents trajectories that are described by the dynamics (2). Next, we analyze the team behavior assuming that the agents have a stable open-loop system matrix. We will show that under this assumption the stability of the error dynamics is guaranteed but as in the float type of fault consensus can no longer be achieved. The following two lemmas summarize our results.

Lemma 5 For the team of agents described in Lemma 1, assume that a follower agent fails to comply with the team control command as specified in (6) and instead implements $u_{f}^{i}=u_{c}$ (LIP fault). The ultimate time varying value of the error vector $e_{s s}$ can be described according to

$$
\begin{aligned}
& e=\left[\begin{array}{l}
e_{w} \\
e_{f}
\end{array}\right] \rightarrow e_{s s} \text { as } t \rightarrow \infty, e_{s s}= \\
& {\left[\begin{array}{c}
-L_{11}^{-1}\left[L_{12} u_{c} t-L_{12} u_{c} t_{f}+L_{12} e^{f}+L_{11}^{-1} L_{12} u_{c}\right] \\
\ldots \ldots \ldots \ldots \ldots \cdots \cdots \cdots \cdots \cdots \cdots \cdots \cdots \cdots \cdots \cdots \cdots \cdots \cdots \\
u_{c} t-u_{c} t_{f}+e^{f}
\end{array}\right]}
\end{aligned}
$$

where $t_{f}$ denotes the time when a fault is injected and $e^{f}$ is the faulty agent velocity error vector at $t_{f}$.

Proof: The proof is omitted due to space limitations.

Lemma 6 a) Stability Analysis: Consider a team of agents with the governing dynamics (1) and the control laws for the followers and the leader given by (10) and (11), respectively. When an agent fails to comply with the designed team control command and instead implements $u_{f}^{i}=u_{c}$, the closedloop error dynamics still remains stable if the corresponding open-loop matrix of the faulty agent, i.e. $A^{f}$, is Hurwitz.

b) Steady State Error: Moreover, the ultimate value of the velocity error vector $e_{s s}$ is governed by

$$
\begin{aligned}
& e=\left[\begin{array}{l}
e_{w} \\
e_{f}
\end{array}\right] \rightarrow e_{s s} \text { as } t \rightarrow \infty, e_{s s}= \\
& {\left[\begin{array}{c}
L_{11}^{-1}\left[L_{12}\left(\left(A^{f}\right)^{-1} B^{f} u_{c}+v^{d}\right)-f_{1}\left(g, v^{d}\right)\right] \\
\ldots \ldots \ldots \ldots \ldots \ldots \ldots \ldots \ldots \ldots \ldots \ldots \ldots \ldots \ldots \\
-\left(A^{f}\right)^{-1} B^{f} u_{c}-v^{d}
\end{array}\right]}
\end{aligned}
$$

where $f_{1}\left(g, v^{d}\right)$ is defined as

$$
f_{1}\left(g, v^{d}\right)=\left[\begin{array}{c}
-\left(K^{1}\right)^{-1}\left(A^{1}\right)^{T}\left(g^{1}+K^{1} v^{d}\right) \\
-\left(K^{i}\right)^{-1}\left(A^{i}\right)^{T} K^{i} v^{d} \\
\vdots \\
-\left(K^{i}\right)^{-1}\left(A^{i}\right)^{T} K^{i} v^{d}
\end{array}\right]
$$

and the superscript $f$ stands for the quantities that correspond to the faulty agent.

Proof: The proof is provided in Appendix A.

\section{Simulation RESUlts}

In this section, simulation results are presented for both LOE and LIP faults that occur in one of the vehicles in a team of four mobile robots. The simulation results corresponding to the float fault are similar to those presented in [6] and are omitted here due to space limitations. Without loss of 
generality the ring topology is considered for the modified leader-follower team.

\section{A. Loss of effectiveness fault}

Simulations are performed for the agents' dynamics as given in (2). The command is assumed to be a pulsedlike signal with a duration of $50 \mathrm{sec}$ and its value switches between $v^{d}=\left[\begin{array}{ll}3 & 4\end{array}\right]^{T}$ and $v^{d}=\left[\begin{array}{ll}5 & -1\end{array}\right]^{T}$. Assuming that the state vector of each agent is given by $X^{i}=\left[\left(r^{i}\right)^{T}, \quad\left(v^{i}\right)^{T}\right]^{T}$, the initial state of the vehicles are selected as $X_{0}^{1}=$ $\left[\begin{array}{llll}6 & 1 & 5 & 3\end{array}\right]^{T}, X_{0}^{2}=\left[\begin{array}{llll}2 & 4 & -5 & -4\end{array}\right]^{T}, X_{0}^{3}=\left[\begin{array}{llll}4 & 3 & -1 & -2\end{array}\right]^{T}$, $X_{0}^{4}=\left[\begin{array}{llll}2 & 0 & 3 & 4\end{array}\right]^{T}$ and the other parameters are selected to be $Q^{i j}=\left[\begin{array}{ll}1 & 0 \\ 0 & 3\end{array}\right], R^{i}=I_{2 \times 2},\left|N^{i}\right|=2$, and $\Gamma=\left[\begin{array}{cc}10 & 0 \\ 0 & 40\end{array}\right]$. Figure 1 shows the actual path trajectories generated by the vehicles in the $x-y$ plane when the fourth agent is injected with a LOE fault during the period $115 \leq t \leq 135$. In this period, the fourth agent's actuator is set to $u_{f}^{i}=.5 u^{i}$. It can be seen that the occurrence of the fault affects the team performance in a short time period and soon after the team recovers its cohesion and achieves consensus. The above verifies the stability and consensus results of the team subject to actuator LOE fault as obtained in Section III.

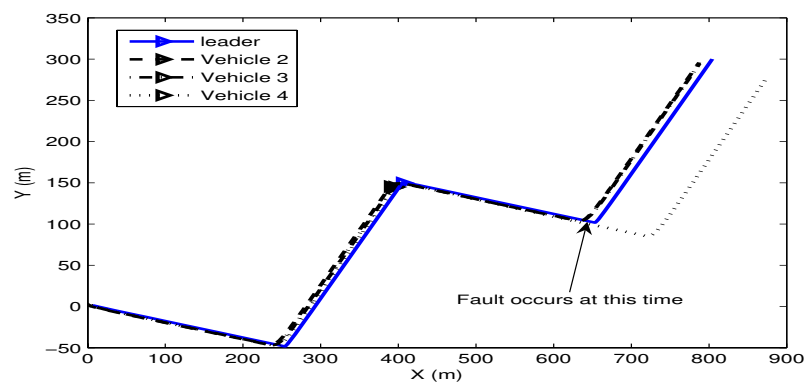

Fig. 1. The $x-y$ path trajectories for the velocity profile of a modified leader-follower (MLF) team of four agents in presence of a LOE fault in the fourth vehicle.

\section{B. Lock-in-place fault}

Simulations are performed for the agents' dynamics as given in (1) and (2). For the latter case, all the settings are the same as the ones used in the case of LOE fault except the command pulse duration which is selected as $20 \mathrm{sec}$. Figure 2 shows the $x$ component of the velocity profile of the agents when the third agent is injected with a LIP fault during the period $20.5 \leq t \leq 25$. During this period, the third agent's actuator is set to $u_{f}^{i}=u^{i}(t=20.5)$. It can be seen that after the occurrence of the fault the agents' velocity diverge to different values as predicted by Lemma 5 .

For the linear dynamical model (1), the command is similar to the previous case and the initial state of the vehicles are the same as before. Other parameters are selected to be $Q^{i j}=100 I_{2 \times 2}$, and $R^{i}=0.01 I_{2 \times 2}$ for $i=2, \ldots, N$. The parameters corresponding to the model are chosen as $\bar{A}^{i}=\left[\begin{array}{ll}1 & 0 \\ 2 & 6\end{array}\right], A^{i}=-I_{2 \times 2}$, and $B^{i}=$

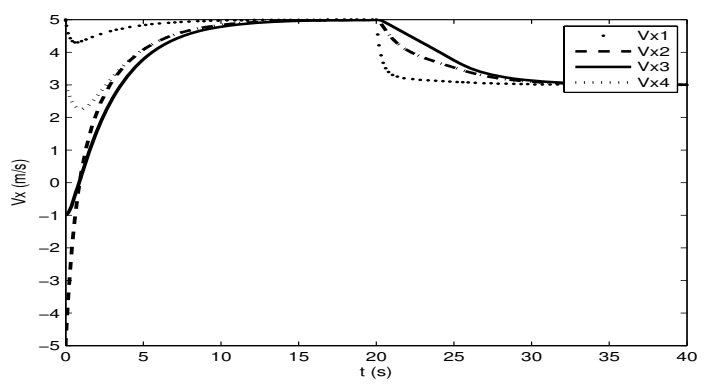

Fig. 2. The $x$ component of the velocity profile of a modified leaderfollower (MLF) team of four agents in presence of a LIP fault in the third vehicle.

$\left[\begin{array}{ll}1 & 1 \\ 2 & 6\end{array}\right]$. The matrices $R^{1}, K^{1}, \Gamma$ are found by solving the set of LMIs in (12) as: $R^{1}=10^{5}\left[\begin{array}{cc}.10 & .17 \\ .17 & 1.27\end{array}\right], K^{1}=$ $10^{4}\left[\begin{array}{cc}8.35 & -1.22 \\ -1.22 & 7.09\end{array}\right]$, and $\Gamma=10^{5}\left[\begin{array}{cc}2.35 & 0 \\ 0 & 1.27\end{array}\right]$.

Figure 3 shows the actual path trajectories that are generated by the vehicles in the $x-y$ plane and Figure 4 shows the $y$ component of the velocity profile of the agents when the third agent is injected with a LIP fault during the period $20.5 \leq t \leq 25$. During this period, the third agent's actuator is set to $u_{f}^{i}=u^{i}(t=20.5)$. It can be seen that after the occurrence of the fault the agents' velocity converge to values that are different from the set-point but are finite. This verifies the stability and boundedness results of the agents' velocity subject to actuator LIP fault as obtained in Lemma 6.

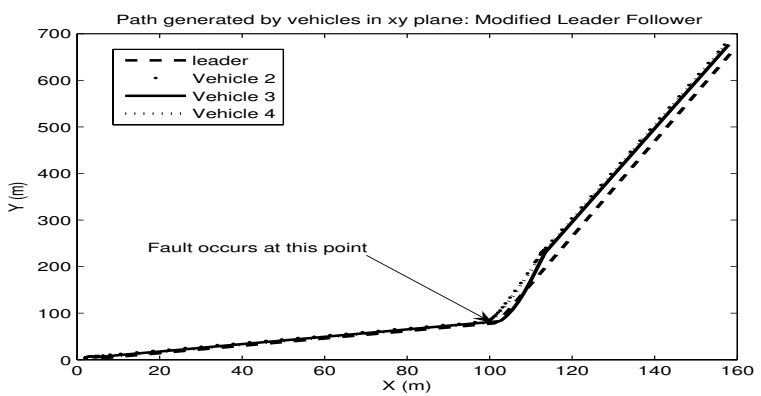

Fig. 3. The $x-y$ path trajectories for the velocity profile of a modified leader-follower (MLF) team of four agents with a linear model in presence of a LIP fault in the third vehicle.

\section{CONCLUSION}

This work provides an analysis and insight into the effects of various actuator faults on the performance of a leaderfollower team of agents. The results are obtained for different types of faults, namely Float, Lock-In-Place (LIP), and Loss of Effectiveness (LOE) that occur in one of the team members. It is shown that the LOE fault does not affect the consensus seeking goal and only results in a transient behavior without any change in the consensus value. 


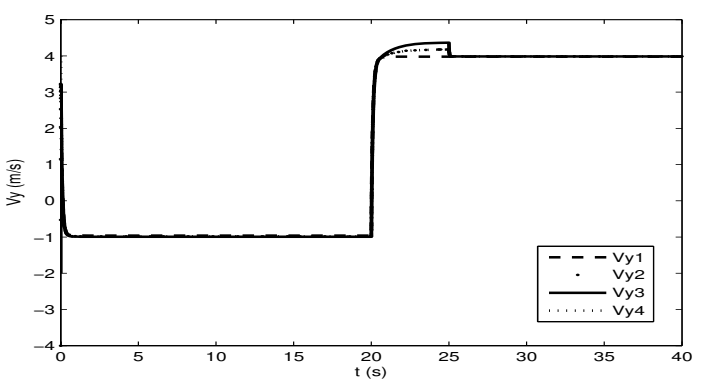

Fig. 4. The $y$ component of the velocity profile of a modified leaderfollower (MLF) team of four agents with a linear model in presence of a LIP fault in the third vehicle.

Moreover, the team remains stable when it is subjected to this type of fault. On the other hand, as shown previously by the authors in [6] when the fault in one or more of the agents is of the float type, the team does no longer maintain its consensus but stability of the team can still be guaranteed and the leader adapts itself to the followers. We have formally shown this adaptability property for the healthy followers. Under the scenario of a LIP fault, team stability can still be guaranteed provided that the open-loop matrix of the faulty agent is stable. Future extensions of this work would be on design of compensating algorithms that avoid deterioration of the team performance in presence of members' faults.

\section{APPENDIX A - PROOF OF LEMMA 6}

Fact 7 For any positive definite (PD) matrix $B$ and matrix A satisfying $A^{T}+A<0\left(A^{T}+A \leqq 0\right)$, the product $C=B A$ is Hurwitz.

According to the discussion given in [12], the error dynamics for the entire team can be written as $\dot{e}=L_{c l} e+f\left(v^{d}, g^{1}\right)$, where $L_{c l}$ is defined as

$$
L_{c l}=-K^{-1}\left(2 L \otimes Q^{i j}+A^{T} K+2 G\right)=-K^{-1} \Upsilon
$$

and $K, A, G, \Upsilon$ are defined in Lemma 2 and $f\left(g^{1}, v^{d}\right)$ is defined by

$$
\begin{gathered}
f\left(g^{1}, v^{d}\right)=\left[\begin{array}{c}
f_{1}\left(g^{1}, v^{d}\right) \\
\ldots \ldots \ldots \\
f_{2}\left(g^{1}, v^{d}\right)
\end{array}\right]= \\
{\left[\begin{array}{c}
-\left(K^{1}\right)^{-1}\left(A^{1}\right)^{T}\left(g^{1}+K^{1} v^{d}\right) \\
-\left(K^{i}\right)^{-1}\left(A^{i}\right)^{T} K^{i} v^{d} \\
\vdots \\
-\left(K^{i}\right)^{-1}\left(A^{i}\right)^{T} K^{i} v^{d} \\
\ldots \ldots \ldots \ldots \ldots \ldots \ldots \\
-\left(K^{f}\right)^{-1}\left(A^{f}\right)^{T} K^{f} v^{d}
\end{array}\right]}
\end{gathered}
$$

When a LIP fault occurs in a follower, the closed-loop error dynamics becomes

$$
\dot{e}=\left[\begin{array}{c}
\dot{e}_{w} \\
\dot{e}_{f}
\end{array}\right]=\left[\begin{array}{ccc}
L_{11} & \vdots & L_{12} \\
\ldots \ldots & \ldots \\
0 & \vdots & A^{f}
\end{array}\right] e+\left[\begin{array}{l}
f_{1}\left(g^{1}, v^{d}\right) \\
A^{f} v^{d}+B^{f} u_{c}
\end{array}\right]
$$

where $L_{i j}$ denotes the corresponding partitioning of the matrix $-K^{-1}\left(2 L \otimes Q^{i j}+A^{T} K+2 G\right)$.

The above dynamical system is stable if both matrices $L_{11}$ and $A^{f}$ are stable. The latter is true by assumption and the former can be shown as follows. Towards this end, we should note that $L_{11}$ can be written as follows

$$
L_{11}=-K_{11}^{-1} \Upsilon_{11}
$$

where $K_{i j}^{-1}, \Upsilon_{i j}$ denote the corresponding partitions of matrices $K^{-1}, \Upsilon$, respectively, which correspond to the faulty and healthy agents dynamics. Now, from Lemma 2 we know that $\Upsilon+\Upsilon^{T}>0$, and therefore its partition $\Upsilon_{11}$ enjoys the same property, i.e. $\Upsilon_{11}+\Upsilon_{11}^{T}>0$. This is due to the fact that $\Upsilon_{11}+\Upsilon_{11}^{T}$ is a principal minor of $\Upsilon+\Upsilon^{T}$ and since $\Upsilon+\Upsilon^{T}$ is $\mathrm{PD}$, any of its principal minors is also PD. Moreover, $K_{11}^{-1}$ is PD for similar reason. Now, invoking Fact 7, we can conclude that $L_{11}$ is stable. Hence, the entire error dynamics is stable. Given that $A^{f}$ is Hurwitz, $\left(A^{f}\right)^{-1}$ is defined and hence in the steady state we have

$$
\left\{\begin{array}{l}
\dot{e}_{f}=A^{f}\left(e_{f}+v^{d}\right)+B^{f} u_{c}=0 \Rightarrow \\
\left(e_{f}\right)_{s s}=-\left(A^{f}\right)^{-1} B^{f} u_{c}-v^{d} \\
\dot{e}_{w}=L_{11} e_{w}+L_{12} e_{f}+f_{1}\left(v^{d}, g^{1}\right) \Rightarrow \\
\left(e_{w}\right)_{s s}=L_{11}^{-1}\left[L_{12}\left(\left(A^{f}\right)^{-1} B^{f} u_{c}+v^{d}\right)-f_{1}\left(v^{d}, g^{1}\right)\right]
\end{array}\right.
$$

This completes the proof of this lemma.

\section{REFERENCES}

[1] R. W. Beard, J. Lawton, and F. Y. Hadaegh, "A feedback architecture for formation control," in Proc. American Control Conference, vol. 6 , June 28-30, 2000, pp. 4087-4091.

[2] R. Olfati-Saber, J. A. Fax, and R. M. Murray, "Consensus and cooperation in networked multi-agent systems," Proc. of the IEEE, vol. 95, no. 1, pp. 215-233, 2007.

[3] F. Xiao and L. Wang, "Consensus problems for high-dimensional multi-agent systems," IET Control Theory Applications, vol. 1, no. 3 , pp. 830-837, 2007.

[4] W. Ren, "Consensus strategies for cooperative control of vehicle formations," IET Control Theory Applications, vol. 1, no. 2, pp. 505$512,2007$.

[5] — "On consensus algorithms for double-integrator dynamics," in Proc. Conference on Decision and Control, Dec. 12-14, 2007, pp. 2295-2300.

[6] E. Semsar-Kazerooni and K. Khorasani, "Optimal performance of a modified leader-follower team of agents with partial availability of leader command and presence of team faults," in Proc. IEEE Conference on Decision and Control, Dec.12-14, 2007, pp. 24912497.

[7] V. Gazi, "Stability of an asynchronous swarm with time-dependent communication links," IEEE Trans. Syst., Man, Cybern. B, vol. 38 , no. 1, pp. 267-274, 2008.

[8] J. D. Bošković, S. M. Li, and R. K. Mehra, "Formation flight control design in the presence of unknown leader commands," in Proc. American Control Conference, May 8-10, 2002, pp. 2854-2859.

[9] E. Semsar and K. Khorasani, "Adaptive formation control of UAVs in the presence of unknown vortex forces and leader commands," in Proc. American Control Conference, June 14-16, 2006, pp. 3563-3568.

[10] E. Semsar-Kazerooni and K. Khorasani, "Optimal consensus algorithms for cooperative team of agents subject to partial information,' Automatica, vol. 44, no. 11, pp. 2766-2777, 2008.

[11] J. D. Bošković, S. E. Bergstrom, and R. K. Mehra, "Retrofit reconfigurable flight control in the presence of control effector damage," in Proc. American Control Conference, June 8-10, 2005, pp. 2652-2657.

[12] E. Semsar-Kazerooni and K. Khorasani, "An optimal cooperation in a team of agents subject to partial information," International journal of Control, vol. 82, no. 3, pp. 571-583, 2009.

[13] R. A. Horn and C. R. Johnson, Matrix Analysis. Cambridge University Press, 1990 